\title{
La diagnostica del diabete insipido
}

\author{
Emanuele Ferrante $^{1}$
}

Accettato: 3 novembre 2020 / Pubblicato online: 8 settembre 2021

(c) The Author(s) 2021

\section{Introduzione}

Il diabete insipido determina la comparsa di poliuriapolidipsia ed è caratterizzato dall'emissione di elevati volumi di urine ipotoniche ( $>50 \mathrm{ml} / \mathrm{kg}$ nelle 24 ore) associata a un incremento dei liquidi assunti per os ( $>3$ litri/die). Se non ben compensata, questa patologia espone principalmente a possibili effetti legati alla disidratazione cellulare (ipotensione, shock) e all'iperosmolalità plasmatica (irritabilità, confusione fino al coma) [1].

\section{Diagnosi di diabete insipido}

La diagnosi differenziale del diabete insipido, dopo l'esclusione delle forme di diuresi osmotica legate in particolare al diabete mellito scompensato e all'ipercalcemia, prevede la distinzione tra: diabete insipido nefrogenico, causato generalmente da una ridotta sensibilità renale all'azione della vasopressina; diabete insipido centrale, causato da una ridotta produzione e secrezione di vasopressina a livello ipotalamo/ipofisario; polidipsia primaria, caratterizzata da un eccessivo introito di liquidi con successiva poliuria compensatoria. Considerato che la terapia delle diverse forme di poliuria-polidipsia è molto differente e che una inappropriata prescrizione espone i pazienti al rischio di sviluppare disordini idro-elettrolitici, è fondamentale eseguire una corretta diagnostica.

Fino a pochi anni fa, la procedura di prima scelta per la diagnosi differenziale era il classico test di assetamento

\footnotetext{
$\triangle$ E. Ferrante

emanuele.ferrante@policlinico.mi.it

1 UOC Endocrinologia, Fondazione IRCCS Ca' Granda, Ospedale Maggiore Policlinico di Milano, Milano, Italia
}

seguito dalla somministrazione endovenosa di desmopressina. Questo test si fonda sulla valutazione indiretta dell'azione della vasopressina mediante la valutazione dell'osmolalità urinaria e i criteri diagnostici utilizzati ancora oggi sono quelli proposti da Miller nel 1970 [2]. Oltre a presentare diversi limiti (lunga durata, impegno personale del paziente e produzione di uno stimolo misto - osmotico e volumetrico - alla produzione di vasopressina), il test di assetamento presenta un'accuratezza diagnostica di circa il 70\%, ulteriormente ridotta in caso di pazienti affetti da polidipsia primaria per via della ridotta capacità renale di concentrare le urine tipica della polidipsia cronica (effetto wash out).

Nonostante questo, le note difficoltà tecniche legate al dosaggio della vasopressina hanno sempre precluso l'utilizzo di un test diretto in questi pazienti. Tali difficoltà sono state recentemente superate in seguito alla disponibilità del dosaggio della copeptina, un glicopeptide di 39 aminoacidi co-secreto in quantità equimolari con la vasopressina, della quale rappresenta un marker surrogato facilmente misurabile e molto stabile [3].

Partendo dal presupposto che un test diretto basato su uno stimolo puramente osmotico potesse rappresentare l'opzione più affidabile, un recente studio prospettico su 156 pazienti con poliuria-polidipsia ha confrontato l'accuratezza diagnostica del test di assetamento classico con quella del dosaggio di copeptina dopo stimolazione osmotica, rappresentata dall'infusione di soluzione salina ipertonica al 3\% allo scopo di portare i livelli di sodio a valori $\geq 150 \mathrm{mEq} / \mathrm{L}$ [4].

Sulla scorta di dati precedenti, questo lavoro ha dapprima confermato che livelli basali di copeptina $>21,4 \mathrm{pmol} / \mathrm{L}$ presentano un'accuratezza diagnostica per diabete insipido nefrogenico del $100 \%$, rendendo di fatto superflue ulteriori procedure. Per quanto riguarda il confronto tra test, il dosaggio diretto di copeptina - utilizzando un cut-off pari a 
Tabella 1 Cut-off diagnostici, accuratezza, SE e SP di differenti test nella diagnosi differenziale della poliuria-polidipsia. $D I$, diabete insipido; DIN, diabete insipido nefrogenico; DIC, diabete insipido centrale; $c D I C$, diabete insipido centrale parziale; $p D I C$, diabete insipido centrale completo; $P P$, polidipsia primaria; Uosm, osmolalità urinaria; $S E$, sensibilità; $S P$, specificità

\begin{tabular}{|c|c|c|c|c|}
\hline & Cut-off diagnostico & Accuratezza & SE & SP \\
\hline \multicolumn{5}{|l|}{ DI nefrogenico vs altre forme di poliuria-polidipsia } \\
\hline Dosaggio di copeptina basale & $\begin{array}{l}\text { Copeptina }>21,4 \mathrm{pmol} / \mathrm{L} \rightarrow \text { DIN } \\
\text { Copeptina }<21,4 \mathrm{pmol} / \mathrm{L} \rightarrow \text { DIC vs PP }\end{array}$ & 100 & 100 & 100 \\
\hline \multicolumn{5}{|l|}{$P P$ vs $D I C$} \\
\hline $\begin{array}{l}\text { Test con soluzione salina ipertonica } 3 \% \text { (dosaggio } \\
\text { di copeptina quando sodio } \geq 150 \mathrm{mEq} / \mathrm{L} \text { ) }\end{array}$ & $\begin{array}{l}\text { Copeptina }>4,9 \mathrm{pmol} / \mathrm{L} \rightarrow \mathrm{DIC} \\
\text { Copeptina }<4,9 \mathrm{pmol} / \mathrm{L} \rightarrow \mathrm{PP}\end{array}$ & $96,5 \%$ & $93,2 \%$ & $100 \%$ \\
\hline $\begin{array}{l}\text { Test con Arginina (dosaggio di copeptina dopo } 60 \\
\text { minuti dall'inizio dell'infusione) }\end{array}$ & $\begin{array}{l}\text { Copeptina }>3,8 \mathrm{pmol} / \mathrm{L} \rightarrow \mathrm{DIC} \\
\text { Copeptina }<3,8 \mathrm{pmol} / \mathrm{L} \rightarrow \mathrm{PP}\end{array}$ & $93 \%$ & $93 \%$ & $92 \%$ \\
\hline $\begin{array}{l}\text { Test di assetamento (Start ore 24, alle ore } 16 \\
\text { iniezione endovena di desmopressina } 2 \mu \mathrm{g} \text { ) }\end{array}$ & $\begin{array}{l}\text { Al termine della fase di assetamento: } \\
\text { 1. UOsm }<300 \mathrm{mOsm} / \mathrm{kg} \\
\rightarrow \text { aumento UOsm 60' dopo DDAVP }>50 \% \rightarrow \text { cDIC } \\
\rightarrow \text { aumento UOsm 60' dopo DDAVP }<50 \% \rightarrow \text { DIN } \\
\text { 2. UOsm 300-800 mOsm } / \mathrm{kg} \\
\rightarrow \text { aumento UOsm 60' dopo DDAVP }>9 \% \rightarrow \text { pDIC } \\
\rightarrow \text { aumento UOsm 60' dopo DDAVP }<9 \% \rightarrow \text { PP }\end{array}$ & $76,6 \%$ & $86,4 \%$ & $69,5 \%$ \\
\hline
\end{tabular}

4,9 pmol/L - dopo infusione di soluzione salina ipertonica al 3\% presentava un' accuratezza diagnostica del 96,5\% nella diagnosi differenziale tra diabete insipido centrale e polidipsia primaria, sensibilmente superiore a quella raggiunta dal test di assetamento $(76,6 \%)$. Lo stesso studio ha mostrato anche che nessuno dei sintomi e/o segni neuroradiologici classicamente utilizzati a supporto diagnostico (in particolare nicturia, comparsa improvvisa dei sintomi, assente iperintensità della neuroipofisi alla risonanza magnetica) presenta una sufficiente specificità nel discriminare tra le diverse forme.

Va sottolineato che il test con soluzione salina ipertonica al 3\% richiede uno stretto monitoraggio del paziente, è gravato da alcuni effetti collaterali (nausea, vertigini, cefalea, malessere) ed è controindicato in soggetti affetti da epilessia, ipertensione non controllata, insufficienza cardiaca classe NYHA III-IV e cirrosi epatica.

Una possibile alternativa è stata recentemente descritta e consiste nella determinazione diretta della copeptina dopo infusione di arginina $(0,5 \mathrm{~g} / \mathrm{Kg}$ in 30 minuti), che rappresenta un potente stimolo non osmotico alla secrezione di vasopressina. Uno studio prospettico su 98 pazienti ha riportato un'accuratezza diagnostica di un singolo dosaggio di copeptina - utilizzando un cut-off pari a 3,8 pmol/L eseguito 60 minuti dopo l'inizio dell'infusione di arginina pari al $93 \%$ [5].

Questo test, di facile gestione, gravato da minori effetti collaterali e per il quale è in corso uno di confronto diretto con il test con ipertonica $3 \%$ su un'ampia popolazione di pazienti, potrebbe in futuro diventare il gold standard per la diagnosi di diabete insipido.

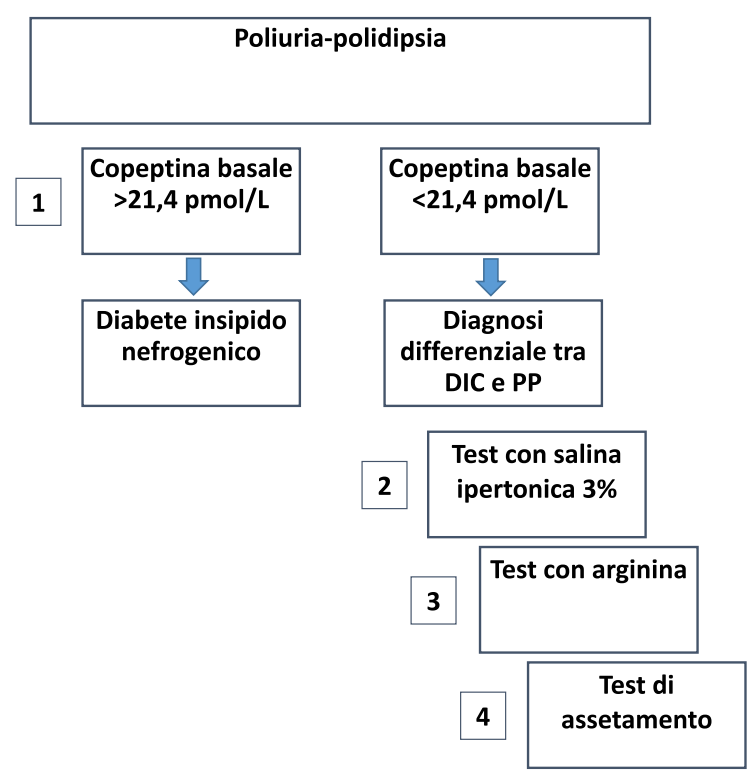

Fig. 1 Proposta di percorso di diagnosi differenziale della poliuriapolidipsia

Le performance diagnostiche dei differenti test utilizzati nella diagnosi differenziale della poliuria-polidipsia sono riassunte nella Tabella 1.

\section{Conclusioni}

La diagnostica del diabete insipido ha subito grandi cambiamenti negli ultimi anni. Attualmente, dopo un'iniziale valutazione basale dei livelli di copeptina al fine di riconoscere le 
forme di diabete insipido nefrogenico, il test di prima scelta è rappresentato dalla determinazione della copeptina stimolata durante infusione di soluzione salina ipertonica al 3\%, seguita dal test con arginina. Il test di assetamento andrebbe invece riservato ai pazienti per i quali non sono percorribili le altre opzioni diagnostiche (Fig. 1).

Funding Note Open access funding provided by Università degli Studi di Milano within the CRUI-CARE Agreement.

Conflitto di interesse L'autore Emanuele Ferrante dichiara di non avere conflitti di interesse.

Consenso informato La studio presentato in questo articolo non ha richiesto sperimentazione umana.

Studi sugli animali L'autore di questo articolo non ha eseguito studi sugli animali.

Nota della casa editrice Springer Nature rimane neutrale in riguardo alle rivendicazioni giurisdizionali nelle mappe pubblicate e nelle affiliazioni istituzionali.

Open Access This article is licensed under a Creative Commons Attribution 4.0 International License, which permits use, sharing, adaptation, distribution and reproduction in any medium or format, as long as you give appropriate credit to the original author(s) and the source, provide a link to the Creative Commons licence, and indicate if changes were made. The images or other third party material in this article are included in the article's Creative Commons licence, unless indicated otherwise in a credit line to the material. If material is not included in the article's Creative Commons licence and your intended use is not permitted by statutory regulation or exceeds the permitted use, you will need to obtain permission directly from the copyright holder. To view a copy of this licence, visit http://creativecommons.org/licenses/by/4.0/.

\section{Bibliografia}

1. Refardt J, Winzeler B, Christ-Crain M (2020) Diabetes insipidus. Endocrinol Metab Clin 49:517-531

2. Miller M, Dalakos T, Moses AM et al (1970) Recognition of partial defects in antidiuretic hormone secretion. Ann Intern Med 73:721-729

3. Morgenthaler NG (2006) Assay for the measurement of copeptin, a stable peptide derived from the precursor of vasopressin. Clin Chem 52:112-119

4. Fenske W, Refardt J, Chifu I et al (2018) A copeptin-based approach in the diagnosis of diabetes insipidus. N Engl J Med 379:428-439

5. Winzeler B, Cesana-Nigro N, Refardt J et al (2019) Argininestimulated copeptin measurements in the differential diagnosis of diabetes insipidus: a prospective diagnostic study. Lancet 394:587-595 